\title{
Author Correction: A surface endogalactanase in Bacteroides thetaiotaomicron confers keystone status for arabinogalactan degradation
}

Alan Cartmell, Jose Muñoz-Muñoz, Jonathon A. Briggs (D), Didier A. Ndeh, Elisabeth C. Lowe, Arnaud Baslé, Nicolas Terrapon (D), Katherine Stott (D), Tiaan Heunis, Joe Gray, Li Yu, Paul Dupree, Pearl Z. Fernandes, Sayali Shah, Spencer J. Williams, Aurore Labourel, Matthias Trost (D), Bernard Henrissat and Harry J. Gilbert (DD

Correction to: Nature Microbiology https://doi.org/10.1038/s41564-018-0258-8, published online 22 October 2018.

In the version of this Article originally published, we reported the protein BT3679 to be an $\alpha$-L-arabinofuranosidase. Recent studies have shown that BT3679 has no demonstrable catalytic activity and thus remains a protein of unknown function. This error was detected in the laboratory of Gideon Davies, at the University of York, who was attempting to obtain the crystal structure of BT3679 in complex with appropriate ligands. His group generated BT3679 independently of our work, and showed that the protein displayed no $\alpha$-L-arabinofuranosidase activity. Once alerted to this possibility, we explored the plasmid population in the master stock of BT3679. Transforming the stock into Escherichia coli and sequencing the plasmid in five single colonies showed that the stock contained two plasmids: BT3679 and BT0348. The BT0348 plasmid was previously shown to encode a GH51 $\alpha$-L-arabinofuranosidase (Nat. Microbiol. 3 , 210-119; 2017). Thus, the reported $\alpha$-L-arabinofuranosidase activity comprised BT0348, but not BT3679. This was confirmed by the Davies laboratory when they analysed a sample of the protein, purified by immobilized metal affinity chromatography from $E$. coli containing the contaminated BT3679 plasmid preparation; they showed that an $\alpha$-L-arabinofuranosidase probe labelled a $51 \mathrm{kDa}$ protein corresponding to the size of the BT0348 plasmid, but not to the $47 \mathrm{kDa}$ BT3679 band.

The Article has thus been amended as follows: (1) Information regarding BT3679 has been removed from the degradative model shown in Fig. 1a,b. Supplementary Fig. 7b,c sub-figures have been removed, and panels referring to BT3679 have been removed from Supplementary Fig. 8; the respective captions have been amended to reflect such changes. Information regarding BT3679 has also been removed from Supplementary Table 2. (2) The designation of BT3679 as a protein of no detectable activity has been stated in Fig. 1c and Supplementary Table 1. (3) Other text referring to BT3679 has been deleted from the Article. 
a

\section{Original}

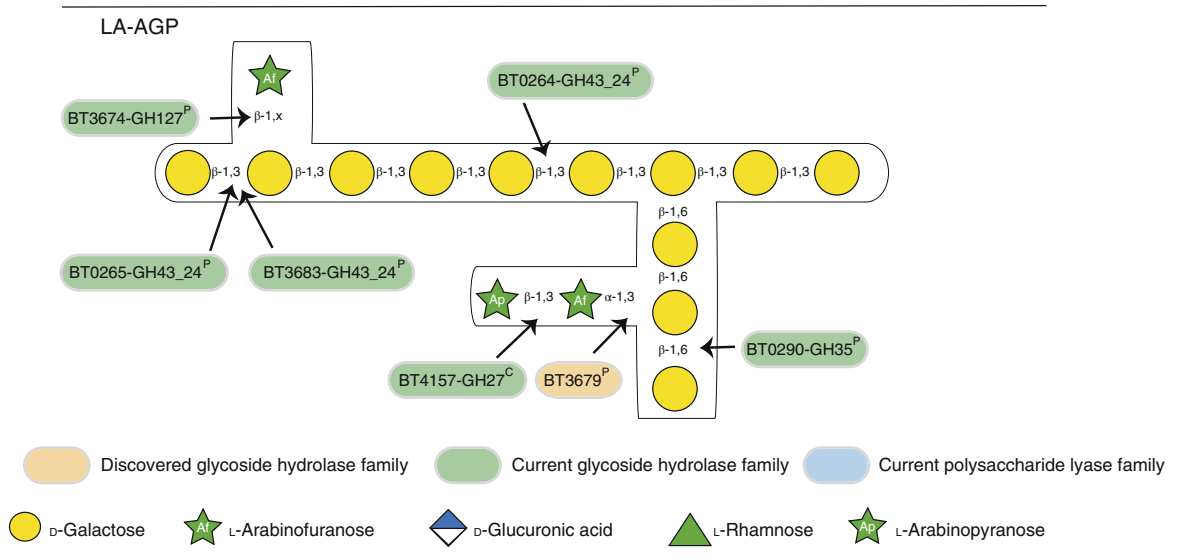

b

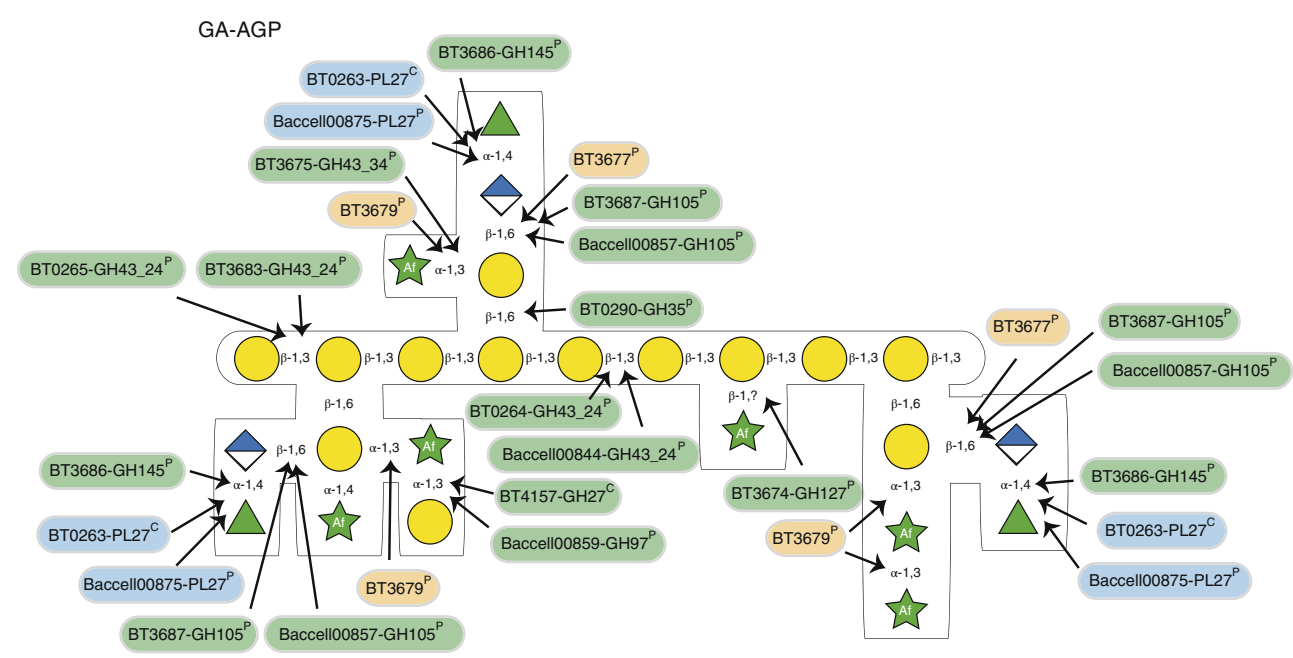

${ }^{\mathrm{C}}$ Cytosolic location ${ }^{\mathrm{P} P e r i p l a s m i c ~ l o c a t i o n ~}$

c

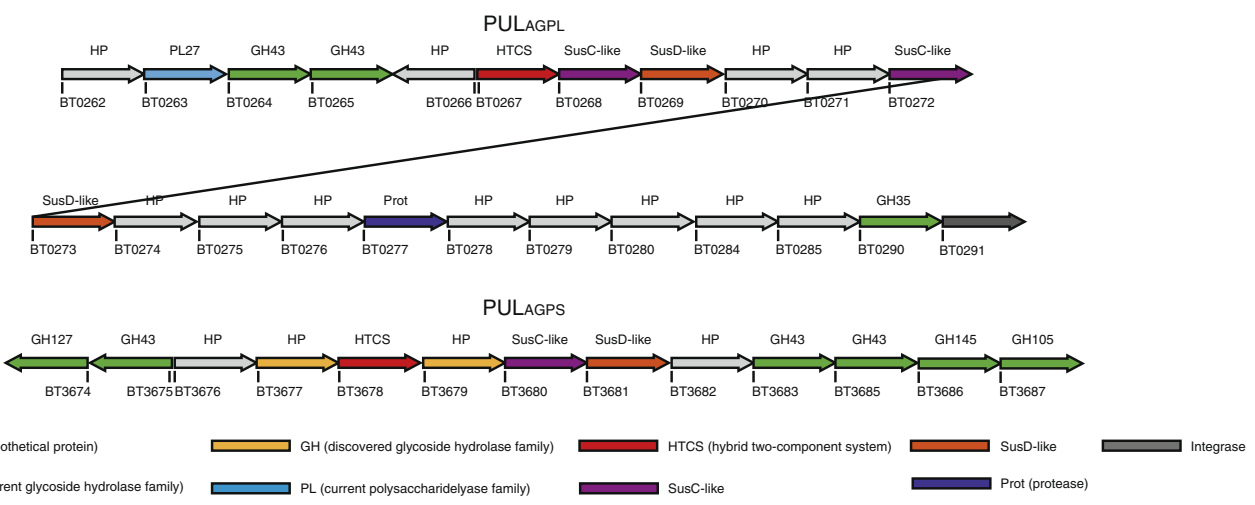

Fig. 1 | Original and Corrected. 


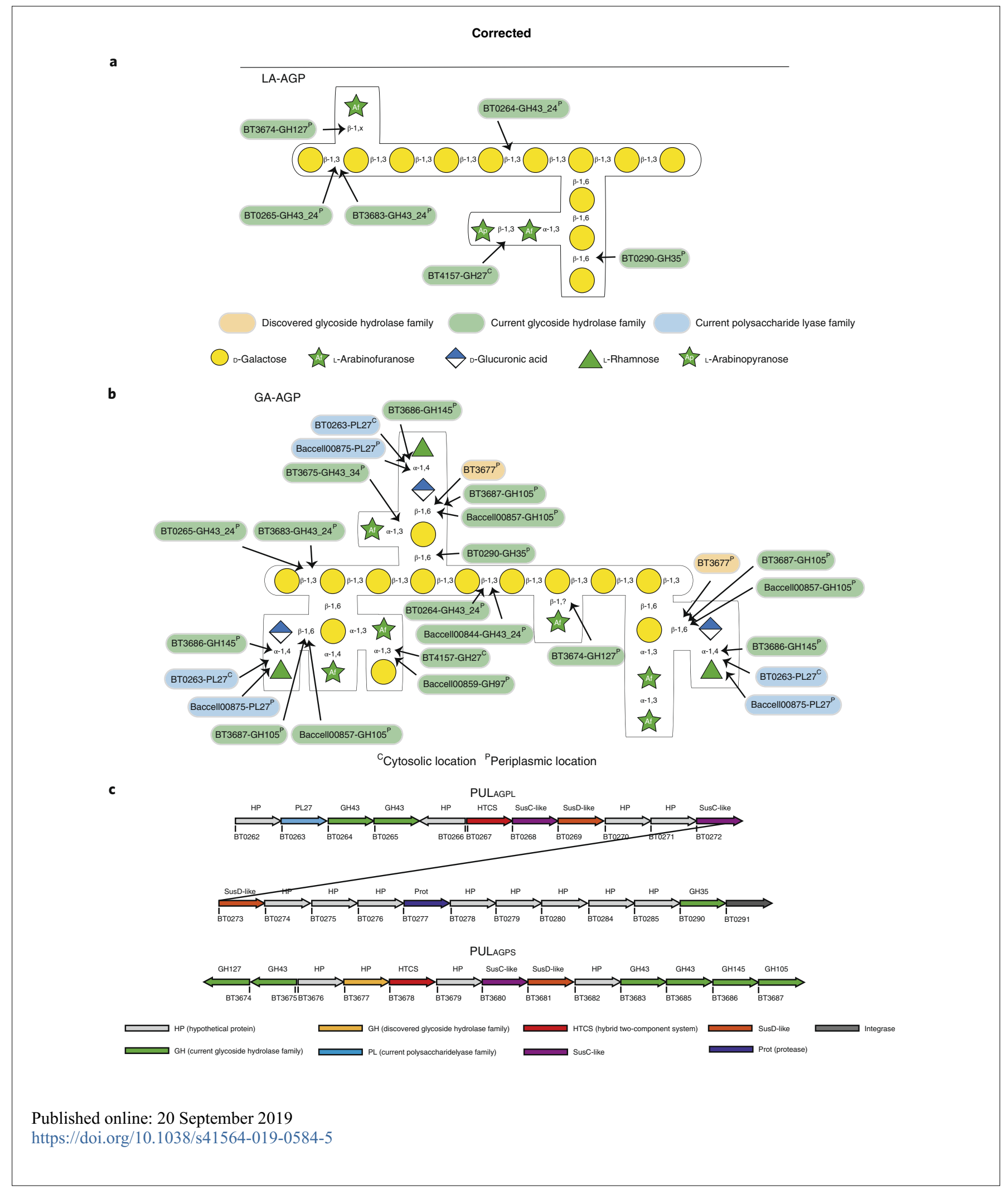

